\title{
Estudios electrofisiológicos, efectividad real de ablación en arritmias y sus complicaciones en Antofagasta como centro de derivación de la segunda región de Chile
}

\author{
Boris Kuzmicic C1, Guillermo Castro G2a, Felipe Gómez N2a, Carolina Espinoza P2b. \\ 1 Electrofisiólogo de la Clínica Antofagasta \\ 2a Interno de Medicina, Universidad de Antofagasta \\ 2b Alumna de Medicina, Universidad de Antofagasta \\ Unidad de Arritmias, Clínica Antofagasta, Antofagasta, Chile.
}

\section{Resumen}

Objetivos: Describir la distribución de las arritmias referidas a un centro privado de electrofisiología de la segunda región, conocer la efectividad inmediata de la terapia ablativa, su rendimiento según las diferentes etiologías y las complicaciones más frecuentemente observadas.

Materiales y Métodos: Se realizó un análisis retrospectivo de los estudios electrofisiológicos (EEF) realizados en Antofagasta entre 1996 y 2007. Se incluyeron 616 pacientes, 321 hombres y 295 mujeres, cuyas edades fluctuaron entre 11 y 91 . El promedio fue 49 años. A cada paciente se le consignó, la indicación de EEF, si fue o no ablativo, y la conclusión.

Resultados: Las indicaciones de EEF fueron en 45\% de los casos Taquicardias Paroxísticas Supraventriculares (TPSV), seguidos por un 14,3\% de Flutter Auricular (FF), un 14,3\% de Síncope y/o trastornos de la conducción, un 11,5\% por Taquicardia Ventricular (TV), 2,9\% por Enfermedad del Nodo, un 1,8\% por Síndrome de Brugada y 10,2\% otras indicaciones. De 616 pacientes, 408 de ellos se realizó terapia de Ablación por radiofrecuencia (ARF), el 88,9\% fue efectivo, 4,2\% parcialmente efectivo (PE) y 6,9\% no efectivos (NE). La efectividad (E) de ARF según etiología fue 93,3\% la Vía Lenta Anterógrada (VLA), 93,3\% Flutter Istmo dependiente, 92,2\% Vías accesorias (VA) y $80,7 \%$ otras etiologías. Del 6,9\% NE el 2,7\% fue FF, 0,99\% vía accesoria lateral izquierda (VALI), 0,74\% taquicardias auriculares, 0,74\% TV, y 1,73\% misceláneas. Las complicaciones fueron perforaciones de cavidades derechas (0,3\%), adenitis inguinal (0,15\%). No existió bloqueo atrioventricular ni mortalidad.

Conclusión: La principal indicación fue TPSV. El procedimiento resultó altamente eficaz, siendo la VLA la con mayor efectividad. La menor E fue FF. Complicaciones menores $0.5 \%$ y mortalidad fue nula.

Palabras Clave: Estudio electrofisiológico, Ablación, Arritmia

\section{Results and complications of electrophysiologic studies and arrhythmia ablation in Antofagasta, Chile}

Background: electrophysiologic studies and ablation therapy is becoming more frequent in Chile. Aim: to describe the distribution of arrhythmias referred to a private electrophysiology center in Antofagasta, to assess immediate efficacy of ablation therapy and incidence of complications.

Correspondencia: Dr. Boris Kuzmicic Calderón

Unidad de Arritmias, Clínica Antofagasta, Antofagasta, Chile.

Calle Matta 1868 Oficina 1009, Antofagasta, Chile

Fono: (9) 8860819 - (55) 410431

Correo Electrónico: bkuzmicic@vtr.net 
Methods: We performed a retrospective analysis of the electrophysiology studies performed in Antofagasta between 1996 and 2007, including 616 patients, 321 men and 295 women, with age range between 11 and 91 years old, (average 49). In every patient, the indication for electrophysiological study, whether or not ablation was performed, and the results were recorded.

Results: Paroxysmal Supraventricular Tachycardia was the indication for $45 \%$ of electrophysiological studies. $14.3 \%$ were cases of Atrial Flutter, $14.3 \%$ of Syncope and/or Conduction disorders, $11.5 \%$ of Ventricular Tachycardia, $2.9 \%$ of Sinus Node Disease, 1.8\% of Brugada's Syndrome and 10.2\% corresponded to other indications. Of 616 patients, 408 were submitted to radiofrequency ablation therapy. This was effective in $88.9 \%$, partially effective in $4.2 \%$ and failed in $6.9 \%$. The effectiveness of radiofrequency ablation according to etiology was $96.5 \%$ for Antegrade Slow Pathway cases, 93.3\% in Isthmus-dependent Flutter, 93.2\% for Accessory Pathway ablation and $80.7 \%$ in other etiologies. The $6.9 \%$ failed cases corresponded to Flutter (2.6\%), Left Lateral Accessory Pathway (0,99\%), Auricular Tachycardia (0.74\%), Ventricular Tachycardia $(0.74 \%)$, and miscellaneous $(1.73 \%)$. The complications were perforation of right cavities $(0,3 \%)$ and inguinal adenitis $(0,15 \%)$. There was no mortality or atrioventricular block.

Conclusion: The main indication for radiofrequency ablation in Antofagasta was Paroxysmal Supraventricular Tachycardia. The procedure turned out to be highly effective, especially in Antegrade Slow Pathway ablation. The less effective procedures included Atrial Flutter. Complication rate was very low.

Key Words: electrophysiology center, ablation therapy

Recibido el 28 de abril de 2009. Aceptado el 16 de junio de 2009

Rev Chil Cardiol 2009; 28: 185-192

\section{Introducción}

En Antofagasta, al igual que en el resto de Chile y del mundo, las arritmias significan una causa no despreciable de morbilidad y mortalidad. Actualmente la terapia por ablación con radiofrecuencia ha reemplazado a la fármaco-terapia con drogas antiarrítmicas en el tratamiento de diversos tipos de arritmias cardíacas. Numerosos han sido los estudios que han demostrado que la terapia por ablación con radiofrecuencia es el tratamiento de primera línea para evitar la recurrencia de eventos 1,3 , como también en los casos en que la patología es latente y pesquisable con anterioridad (Wolf Parkinson White) siendo la opción terapéutica de elección para evitar la aparición de un primer episodio2,3,4.

Durante las últimas décadas, los estudios electrofisiológicos se han transformado en instrumentos clínicos extensamente usados, a menudo indispensables, en la evaluación de pacientes con arritmias cardíacas específicas ${ }^{3}$.

Como tales estudios llevan un riesgo relativamente pequeño, pero finito de complicaciones, consumen recursos de atención en salud, además dado que rutinariamente implican la inducción de serias arritmias durante los procedimientos, es importante que su utilidad clínica para el diagnóstico y la terapia de arritmias cardíacas sea considerada cuidadosamente $3,4,5$.

Entre las ventajas de la ablación por radiofrecuencia se pueden incluir el alivio de la sintomatología, la eliminación de la necesidad de terapias antiarrítmicas de por vida, y el ahorro de costos en medicamentos a largo plazo, produciendo una mejora de la capacidad funcional y por lo tanto en su calidad de vida. La principal desventaja es el riesgo de complicaciones, que varía según el tipo de procedimiento de ablación y la experiencia y habilidad del operador y su equipo de trabajo. Por lo tanto, la proporción riesgo-beneficio para la ablación con radiofrecuencia debería ser siempre considerada sobre una base individual antes de que el procedimiento sea realizado 1,5 .

Los resultados varían significativamente en los 
distintos centros y según los distintos operadores 2,3 .

Habitualmente tendemos a utilizar como referencia, para la toma de decisiones y comparación de resultados, publicaciones internacionales que muchas veces no son comparables en cuanto a números de procedimientos, tecnología y capacitación profesional, en relación a nuestro ambiente local, dado más que nada por la poca publicación local de estudios electrofisiológicos.

En el presente estudio, pretendemos describir la realidad de un centro privado de derivación de electrofisiología a nivel regional, en forma representativa de la actividad de otros centros nacionales con características similares. En el cual revisamos principalmente el motivo de consulta de los afectados, la tasa de respuesta del tratamiento y las vías implicadas, así como la prevalencia de los trastornos del ritmo, y ver como se ajusta ésta experiencia a la descrita por la bibliografía relacionada al tema. Otro punto será el revisar la incidencia de complicaciones asociadas a los procedimientos $y$, a la vez, compararlas dentro de lo posible a lo descrito.

\section{Métodos}

Se realizó un análisis retrospectivo de los estudios electrofisiológicos (EEF) realizados en la Unidad de Arritmias de la Clínica Antofagasta entre los años 1996 y 2007.

Los estudios electrofisiológicos fueron realizados con un equipo de electrofisiología modelo EP-Tracer de Cardiotek ${ }^{\circledR}$, un Angiógrafo Philips ${ }^{\circledR}$ Diagnost C2, y un generador de radiofrecuencia Atakr I y II de Medtronic ${ }^{\circledR}$. Se utilizó anestesia local y bajo técnica de Seldinger se procedió a puncionar vena y/o arteria femoral. Por éstas vías se introducen electrocatéteres: bipolares o cuadripolares que se dejan en la aurícula derecha y en la región del haz de His. Además de un catéter de ablación habitualmente Conductr o Marinr MC o XL (Medtronic ${ }^{\circledR}$ ) con oliva distal de 4, 5 u $8 \mathrm{~mm}$ que se deja en el seno coronario o para mapeo endocavitario derecho o izquierdo.

El estudio se realizó con las fichas recolectadas en la unidad de arritmias de la clínica Antofagasta entre los años 1996 y 2007. Los datos fueron tabulados utilizando plantilla Excel 2003. Fueron incluidos 616 pacientes, como criterio de selección del paciente se requería haber realizado un estudio electrofisiológico, y no se consideró si el paciente había sido ingresado a una reablación. A cada paciente se le consignó la indicación por la cual se envió para estudio electrofisiológico, la arritmia que el paciente presentaba al momento del examen, si este examen fue ablativo o no, qué tiempo tuvo de exposición a Rayos $\mathrm{X}$, qué tipo de vía fue ablacionada y la conclusión. Se denominó como ablación por radiofrecuencia efectiva a la supresión completa de la inducibilidad de la arritmia debido a la destrucción de su sustrato anatómico funcional. Por ejemplo a la completa eliminación de una vía accesoria.

Se consideró como ablación parcialmente efectiva a la modificación significativa del sustrato anatómico funcional de la arritmia, sin llegar a conseguir su definitiva eliminación, siendo ésta suficiente para impedir la re inducción de la arritmia clínica. Un ejemplo de esto es la persistencia de la inducibilidad de algunas ecos nodales sucesivos sin lograr la re inducción de una reentrada nodal sostenida, tanto en condiciones basales como durante la infusión de Isoproterenol ${ }^{\circledR}$.

\section{Resultados}

Se seleccionaron un total de 616 pacientes, de los cuales 321 fueron hombres y 295 mujeres, sus edades fluctuaron entre 11 y 91 años, siendo el promedio los 49 años de edad. Las indicaciones para realizar los estudios electrofisiológicos (Gráfico 1) fueron en un $45 \%$ de los casos Taquicardias Paroxísticas Supraventriculares, seguidos por un $14,3 \%$ de Flutter Auricular, un 14,3\% de Síncope y/o trastornos de la conducción, un 11,5\% por Taquicardia Ventricular, 2,9\% por Enfermedad del Nodo, un 1,8\% por Síndrome de Brugada y $10,2 \%$ otras indicaciones. De 616 pacientes, en 408 de ellos se realizó terapia de ablación por radiofrecuencia, de los cuales $363(88,9 \%)$ fueron efectivos, $17(4,2 \%)$ parcialmente efectivos y $28(6,9 \%)$ no efectivos (Gráfico 2).

La efectividad de la ablación por radiofrecuencia según sus vías (Gráfico 3) fueron de 93,3\% para la Vía Lenta Anterógrada, 93,3\% el Flutter Istmo dependiente, $93,2 \%$ Vías accesorias y otras etiologías $80,7 \%$. Del 
6,9\% de las ablaciones no efectivas (Gráfico 4) el 2,7\% correspondió a Flutter Auricular, 0,99\% a Vía Accesoria Lateral Izquierda, 0,74\% a Taquicardias Auriculares, $0,74 \%$ fueron Taquicardias Ventriculares, y $1,73 \%$ misceláneas. Dentro de las complicaciones de los estudios electrofisiológicos (Gráfico 5 ) se presentaron 2 perforaciones de cavidades derechas $(0,3 \%), 1$ adenitis inguinal $(0,15 \%)$, y no se presentó ningún bloqueo atrio ventricular iatrogénico, no existiendo casos de mortalidad. En la tabla 1 (Gráfico 6) se puede ver la efectividad según su tipo de arritmia, su $\mathrm{N}$, su efectividad y sus complicaciones. El tiempo promedio de exposición a Rayos X fue 20,5 minutos, siendo el tiempo mínimo de exposición 2 minutos y el máximo 77 minutos, independiente si el estudio electrofisiológico realizado fue ablativo o no.
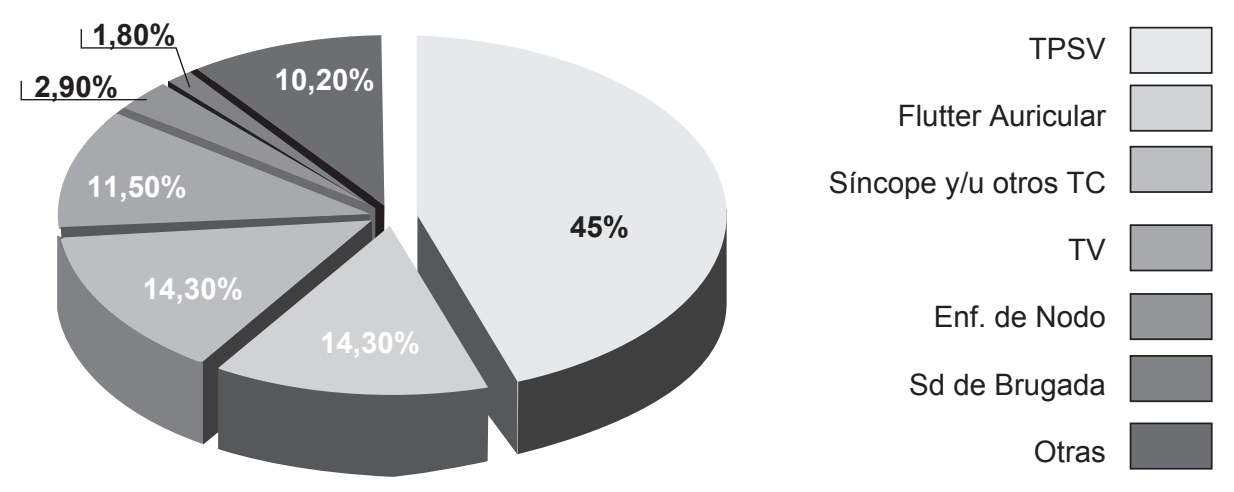

Gráfico1: Indicaciones de Estudio electrofisiológico.

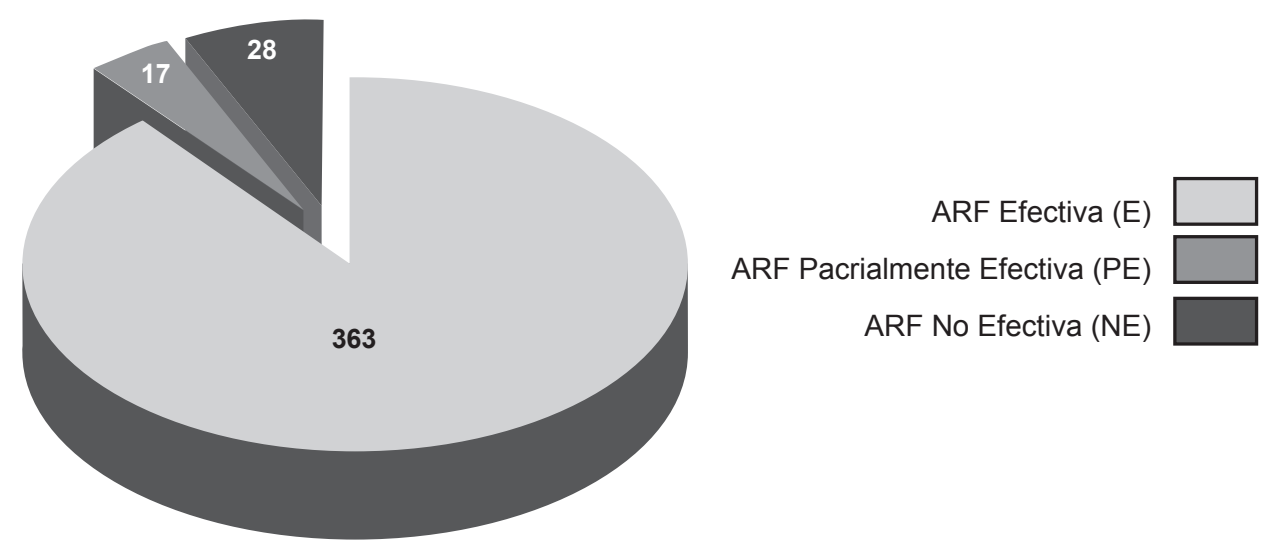

Gráfico 2: Efectividad sobre el total de EFE realizados. 
Estudios electrofisiológicos, efectividad real de ablación en arritmias y sus complicaciones en Antofagasta...

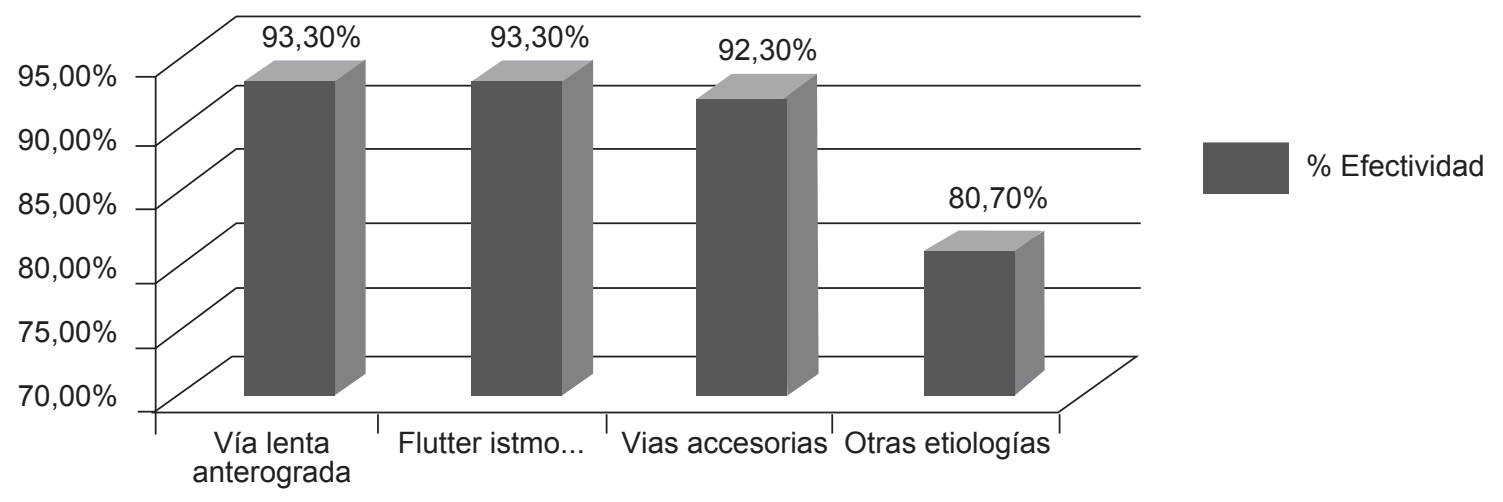

Gráfico 3: Porcentaje de efectividad según vía ablacionada.

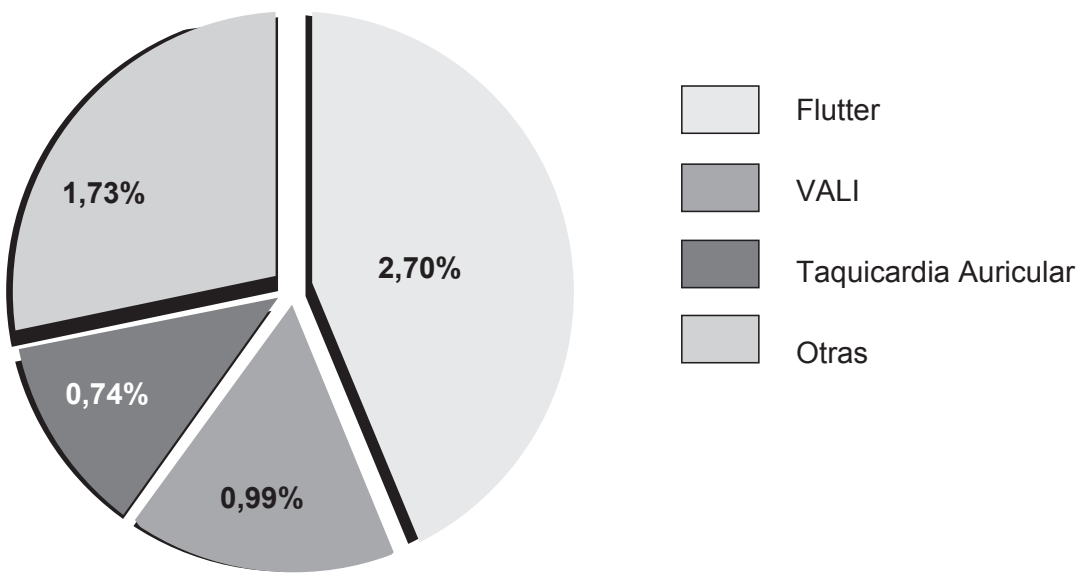

Gráfico 4: Desglose de las Ablaciones no efectivas.

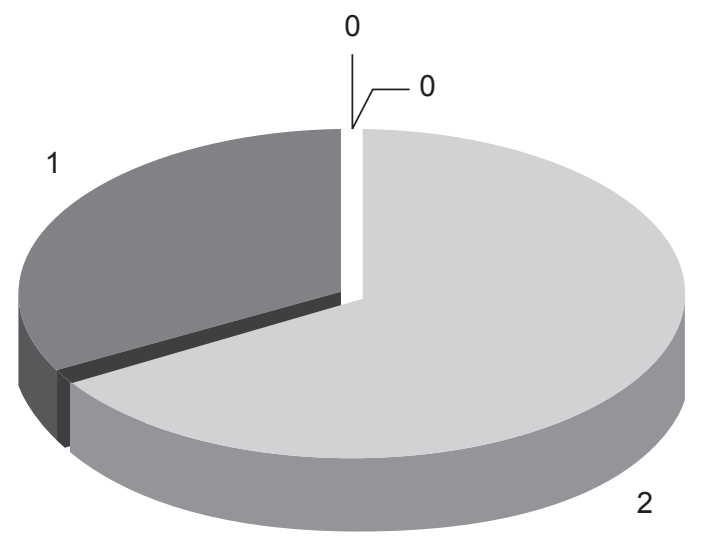

Perf Cav Derechas

Adenitis Inguinal

BAV latrogénico

Muertes

Gráfico 5: № Absoluto de complicaciones por EEF 


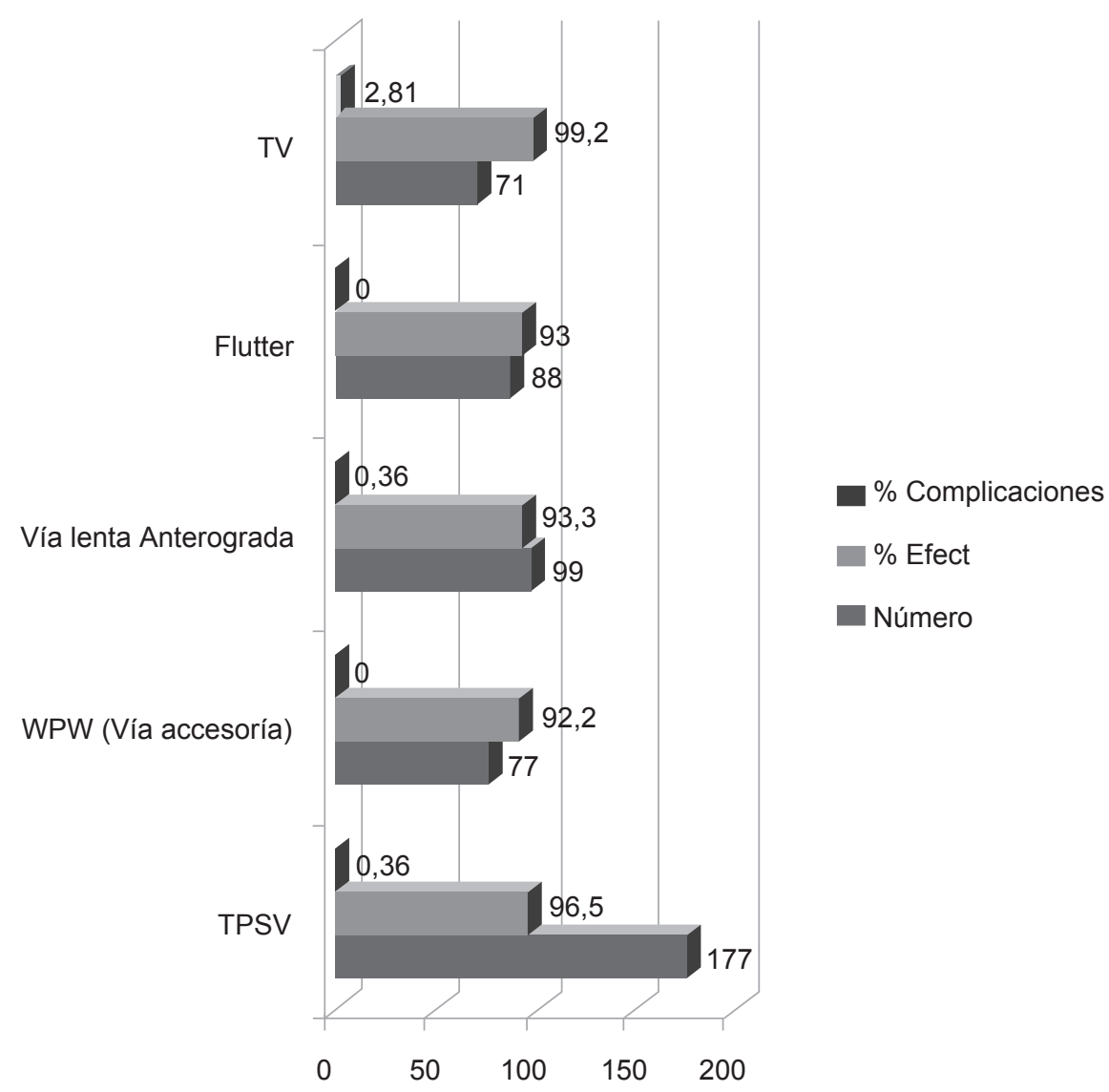

Gráfico 6: Tipo de Arritmia, su efectividad y complicaciones.

\section{Discusión}

La prevalencia de la consulta de pacientes a servicios de urgencia o a consultas privadas por arritmias o manifestaciones indirectas de éstas, hace necesario conocer la epidemiología general de su presentación, tanto etiológica, como semiológica. Variados estudios han tratado la temática de la semiología asociada a estos eventos, dejando ahora en sobre mesa el qué pasa cuando estos pacientes son derivados. ¿Son estos tratamientos efectivos?, ¿vale la pena arriesgarse a un procedimiento invasivo? Los estudios generalmente demuestran altas tasas de efectividad en centros especializados $4,5,6,7$ y es por esto que nosotros evaluamos la efectividad de la terapia radio-ablativa en un centro privado, determinando si los datos presentados en otras publicaciones son validables en nuestra realidad.

Los resultados obtenidos en este estudio se ajustan estadísticamente a lo mostrado por el "Multicentric European Radiofrequency Survey" (MERFS) en relación a la efectividad global de las terapias ablativas, con una inefectividad de alrededor de un $4,7 \%$ contra un $6,9 \%$ global en el presente estudio, así como a la prevalencia de complicaciones 9,14 .

Sin embargo los análisis en relación a las etiologías en algunos aspectos no son comparables, ya que muchas de las categorías analizadas en este trabajo agrupadas como "Otras" son analizadas individualmente, o subdividen los distintos tipos de vías accesorias 8,10 , que nosotros agrupamos intentando simplificar los análisis ${ }^{11}$, por lo que muchos de las sub categorías no son comparables. Final- 
mente la tasa global de complicaciones la mortalidad en nuestro centro fue de $0 \%$, siempre considerando una casuística inferior a lo analizado en los grandes estudios multicéntricos y la tasa de complicaciones no mortales 12,13 , se asemeja a los vistos en el estudio MERFS, al que nos referimos anteriormente. Todo esto hace a la terapia de ablación por radiofrecuencia un procedimiento con una efectividad comprobada y segura 14,15 , por lo tanto, un procedimiento recomendable como primera línea en la resolución de patologías eléctricas del corazón, pudiendo ofrecer esta terapia a los pacientes, sabiendo que un profesional con experiencia no tiene mayores complicaciones $3,6,10,11,12$. La principal indicación de estudios electrofisiológicos fue la Taquicardia Paroxística Supraventricular6,7,15. El procedimiento de ablación por radiofrecuencia resultó altamente eficaz, siendo la Vía Lenta Anterógrada la con mayor efectividad al ser ablacionada. Sin embargo, hay que destacar que la etiología con menor efectividad fue el Flutter Auricular. La inefectividad observada en alguna de las vías accesorias laterales izquierdas pudiera explicarse por su abordaje vía Aórtica retrógrada, no realizándose punción transeptal en la misma sesión de ablación para complementar la ablación de la inserción atrial de la vía accesoria cuando la ablación del aspecto ventricular del haz no logra interrumpir la conducción 16,17,18.

Cabe mencionar que en el presente trabajo queda la duda de las tasas de recurrencia real, y efectividad a corto y mediano plazo por falta de seguimiento de los pacientes. Este tema podría ser analizado en una futura investigación.

\section{Agradecimientos:}

A todo el personal del laboratorio de electrofisiología de la Clínica Antofagasta, que estuvieron con nosotros a lo largo de estos 12 años, ya que sin su colaboración no sería posible la realización de este arduo trabajo diario.

A Carolina Ureta Raboy, E.U. Asistente de Servicios Biomédicos de Centro Saval por el aporte de información indispensable para la realización del trabajo.

\section{Referencias}

1. MORADY F, Radio frequency ablation as treatmen for cardiac arrhythmias N Engl J Med 1999; 340:541.

2. CALKINS H, YOUNG P, MILLER J, OLSHANSKY B, CARLSON M, SAUL J,et al. Catheter ablation of accessory pathways, atrioventricular nodal reentrant tachycardia, and the atrioventricular junction.

Circulation 1999;99:2 62-70

3. ZIPES D,DIMARCO J, GILLETTE P, JACKMAN W, MYERBURG R, RAHIMTOOLA S. Guidelines for clinical intracardiac electrophysiological and catheter ablation procedures. JACC 1995; 26: 556-560

4. HELGUERA M, DE ELIZALDE G, MAID G, CORRADO G, CAGIDE A, DOVAL $\mathrm{H}$, et al. Ablacion por radiofrecuencia para el tratamiento de las arritmias cardiacas Rev. Arg de cardiología 2003; 71:402-407

5. PAPPONE C, SANTINELLI V, MANGUSO F,AUGELLO G, SANTINELLI O, VICEDOMINI G, et al. A Randomized Study of Prophylactic Catheter Ablation in Asymptomatic Patients with the Wolff-Parkinson-White Syndrome N Engl J Med 2003; 349:1803-11.

6. DELACRETAZ E, Supraventricular Tachycardia, N Engl J Med 2006;354:1039-45.
7. MORADY F, Radio frequency ablation as treatmen for cardiac arrhythmias N Engl J Med 1999; 340: 534-544

8. ORAL H, PAPPONE C, CHUGH A, GOOD E, BOGUN F, PELOSI F, et al. Circumferential Pulmonary-Vein Ablation for Chronic Atrial Fibrillation, N Engl J Med 2006;354:934-41.

9. SWARTZ J, TRACY C, FLETCHER R, et al. Radiofrequency endocardial catheter ablation of accessory atrioventricular pathway atrial insertion sites. Circulation 1993;87:487-99.

10. OZCAN C, JAHANGIR A, FRIEDMAN P, PATEL P, MUNGER $T$, REA $R$, et al. Long term survival after ablation of the atrioventricular node and implantation of a permanent pacemaker in patients with atrial fibrillation. $\mathrm{N}$ Engl $\mathrm{J}$ Med, Vol. 344:1043-51

11. PAPPONE C, MANGUSO F,SANTINELLI R, VICEDOMINI G, SALA S, PAGLINO G, et al. Radiofrequency Ablation in Children with Asymptomatic Wolff-Parkinson-White Syndrome. N Engl J Med 2004;351:1197-205.

12. REDDY V,REYNOLDS M, NEUZIL P, RICHARDSON A, TABORSKY M, JONGNARANGSIN K, et al. Prophylactic Catheter Ablation for the Prevention of Defibrillator Therapy. N Engl J Med 2007;357:2657-65.

13. ROY D, TALAJIC M, NATTEL S, WYSE G, DORIAN P, LEE K 
et al. Rhythm Control versus Rate Control for Atrial Fibrillation and Heart Failure. N Engl J Med 2008;358:2667-77.

14. HINDRICKS G, et al. Incidence of complete atrioventricular block following attempted radiofrequency catheter modification of the atrioventricular node in 880 patients Results of the Multicenter European Radiofrequency Survey (MERFS) European Heart Journal 1996;17:82-88

15. CHENG C, SANDERS G, HLATKY M, HEIDENREICH P, MCDONALD K, LEE B, et al. Cost-Effectiveness of Radiofrequency Ablation for Supraventricular Tachycardia. Ann Intern Med. 2000;133:864-876.

16. SILVA R, MONTB L, BERRUEZOB A, FOSCHB X, WAYARB L,
ALVARENGAB $N$, et al. Ablación por radiofrecuencia para el tratamiento de la fibrilación auricular focal a través de cartografía circunferencial y aislamiento segmentario de las venas pulmonares Rev Esp Cardiol 2003;56(4):361-7

17. BUXTON A, CALKINS H, CALLANS D, DIMARCO J, FISHER $J$, GREENE $H$, et al. Key Data Elements and Definitions for Electrophysiological Studies and Procedures ACC/AHA/HRS Clinical Data Standards JACC Vol. 48, No. 11, 2006 December 5, 2006:2360-96

18. THOMAS K, JOSEPHSON M The Role of Electrophysiology Study in Risk Stratification of Sudden Cardiac Death. Progress in Cardiovascular Diseases, 2008; 51:97-105 